\title{
Space localization and uniqueness of solutions of a quasilinear parabolic system arising in semiconductor theory
}

\author{
Jesús Ildefonso DÍAZ, Gonzalo GALIANO and Ansgar JÜNGEL
}

J. I. D.: Departamento de Matemática Aplicada,

Universidad Complutense de Madrid, 28040 Madrid, Spain;

E-mail : jidiaz@sunma4.mat.ucm.es

G. G.: Stichting Methematish Centrum, Kruislaan 413, 1098 SJ Amsterdam, The Netherlands;

E-mail : galiano@ewi.nl

A. J.: Fachbereich Mathematik, Universität Rostock, 18055 Rostock, Germany.

E-mail : jungel@sun.math.uni-rostock.de

\begin{abstract}
A degenerate parabolic system consisting in two continuity equations for densities of charged particles and in the Poisson equation for an electric potential is considered. We show the finite speed of propagation, a waiting time property for the vacuum (null) sets and a property of formation of vacuum. The proofs are based on energy methods. Furthermore, some results on the uniqueness of solutions are proved by using different duality methods.
\end{abstract}

\section{Localisation spatiale et unicité des solutions d'un système parabolique quasi-linéaire dans la théorie des semi-conducteurs}

Résumé. On considère un système parabolique dégénéré consistant en deux équations de continuité pour les densités des particules chargées et en l'équation de Poisson pour le potentiel électrique. On démontre que la vitesse de propagation est finie. On établit une propriété du temps d'attente pour les ensembles du vide et une propriété de formation $d u$ vide. Les démonstrations sont basées sur des méthodes d'énergie. En outre, on montre des résultats d'unicité des solutions en utilisant différentes méthodes de dualité.

\section{Version française abrégée}

On considère le problème (1) modélisant le transport des particules chargées dans un semi-conducteur influencé par un champ électrique. Le modèle est très voisin de ce qui apparaît dans la théorie de la filtration biphasique (voir [1]). Si la fonction $r$ satisfait (2), le problème est de type dégénéré. Les solutions qui satisfont $n=0$ ou $p=0$ localement sont appelées solutions de vide. Le modèle est dérivé

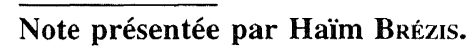




\section{J. 1. Díaz, G. Galiano and A. Jüngel}

d'un modèle de dérive-diffusion pour les semi-conducteurs, basé sur la statistique de Fermi-Dirac (voir [6]). L'existence des solutions satisfaisant (3) a été montrée dans [5].

La première partie de la Note est consacrée à l'étude du comportement des solutions de vide. Si $n(0)=0$ dans une boule $B_{\rho_{\circ}}\left(x_{o}\right) \lessdot \subset \Omega$, et si l'énergie locale de $n$ et $p$ dans le cylindre $B_{\rho_{o}}\left(x_{o}\right) \times(0, T)$ est suffisamment petite, alors $n=0$ et $p=0$ pour $x \in B_{\rho(t)}\left(x_{o}\right), t \in\left(0, T_{1}\right)$, avec $T_{1}>0$ et $\rho(t)$ une certaine fonction décroissante positive. Si on fait de plus l'hypothèse (5) (et on suppose $p>0$ localement), alors $n=0$ dans le cylindre $B_{\rho_{o}}\left(x_{o}\right) \times\left(0, T_{2}\right)$ avec $T_{2}>0$. Le troisième résultat donne l'existence de solutions de vide sous l'hypothèse (5) même si $n(0)$ est globalement positif.

Pour les démonstrations on utilise une méthode d'énergie présentée dans [2]. On commence avec une formule locale d'intégration par parties (7), où $P$ est le domaine d'intégration défini par (6). La nouveauté de nos estimations réside dans le traitement des termes convectifs dans (1). Les estimations de $I_{1}, I_{2}$ et $I_{3}$ sont obtenues comme dans [2]. On emploie un lemme d'interpolation et l'inégalité de Hölder. L'estimation de l'intégrale $I_{4}$ est faite de plusieurs manières. Pour la démonstration du Théorème 1 on prend comme $P$ un cône tronqué. Alors la deuxième intégrale sur la surface latérale de $P$ à droite de (10) est négative pour $-\sigma>0$ suffisamment grand. On prend un cylindre $P$ dans la démonstration du Théorème 2 et un paraboloïde dans le Théorème 3 . Dans ces deux cas le terme convectif dans $I_{4}$ peut être estimé en utilisant le terme $R(n, p) n^{\alpha}$. On arrive à certaines inégalités différentielles (voir, par exemple, (8)) ce qui permet de conclure.

Des résultats d'unicité pour le problème plus général $(\mathcal{P})$ sont présentés dans la deuxième partie de la Note. Le Théorème 4 montre l'unicité sous trois hypothèses distinctes à l'aide de techniques de dualité. Dans le cas de la partie (i), on adapte la technique de [1], dans (ii) le résultat est valide pour $r$ non strictement croissante mais sous la condition (12) (on utilise un argument de [7]). Finalement, dans la partie (iii) on prend une technique inspirée de [3] pour des solutions fortes telles que $n, p \in L^{\infty}\left(0, T ; W^{1, q}(\Omega)\right)(q=1$ si $N=1$ et $q>N$ si $N>1)$ et pour $r \in C^{2}(0, \infty)$ convexe strictement croissante.

\section{The model}

The following initial boundary value problem describes the transport of charged particles in a semiconductor subject to an exterior electric field:

$$
\begin{cases}n_{t}-\nabla \cdot(\nabla r(n)-n \nabla V)=-R(n, p) & \text { in } Q_{T}, \\ p_{t}-\nabla \cdot(\nabla r(p)+p \nabla V)=-R(n, p) & \text { in } Q_{T}, \\ \Delta V=n-p-C & \text { in } Q_{T}, \\ r(n)=r\left(n_{D}\right), \quad r(p)=r\left(p_{D}\right), \quad V=V_{D} & \text { on } \Sigma_{D T}, \\ \nabla r(n) \cdot \nu=\nabla r(p) \cdot \nu=\nabla V \cdot \nu=0 & \text { on } \Sigma_{N T}, \\ n(x, 0)=n_{I}(x), \quad p(x, 0)=p_{I}(x) & \text { in } \Omega .\end{cases}
$$

Here, $n$ is the electron density, $p$ the (positively charged) hole density, $V$ the electric potential, $C=C(x)$ the doping profile of the semiconductor device, and $\nu$ the exterior normal. The recombination and generation of electrons and holes is modelled by $R(n, p)$. The function $r$ satisfies:

$$
r \in \mathcal{C}^{1}(\mathbb{R}), \quad r^{\prime}(0)=0, \quad \text { and } \quad r^{\prime}(s) \geq 0 \text { for any } s \in \mathbb{R},
$$

and can be interpreted as the pressure of the particles. Usually, $r(s):=|s|^{\alpha}$ sign $(s)$ with $\alpha \in(1,5 / 2)$ (see [6]). 
The (bounded) semiconductor domain is denoted by $\Omega \subset \mathbb{R}^{d}, d \leq 3$. Its (regular) boundary consists of two disjoint subsets $\Gamma_{D}$ and $\Gamma_{N}$. In [5] and [4] it is proved that there exists a weak solution $(n, p, v)$ to (1) such that

$$
\begin{aligned}
& 0 \leq n, p \in L^{\infty}\left(Q_{T}\right) \cap H^{1}\left(0, T ; \mathcal{V}^{\prime}\right) \cap \mathcal{C}\left([0, T] ; L^{1}(\Omega)\right), \\
& \nabla r(n), \nabla r(p) \in L^{2}\left(Q_{T}\right), \quad V \in L^{\infty}\left(0, T ; W^{2, p}(\Omega)\right) \quad \forall p<\infty,
\end{aligned}
$$

where $\mathcal{V}^{\prime}$ is the dual space of $\mathcal{V}=\left\{u \in H^{1}(\Omega): u=0\right.$ on $\left.\Gamma_{D}\right\}$ and where it is assumed that the function $R$ satisfies conditions of monotonicity (see [5]) or Lipschitz continuity (see [4]), and $C \in L^{\infty}(\Omega), 0 \leq n_{I}, p_{I} \in L^{\infty}(\Omega), 0 \leq n_{D}, p_{D} \in L^{\infty}\left(Q_{T}\right) \cap H^{1}\left(Q_{T}\right), V_{D} \in L^{\infty}\left(0, T ; W^{2, \infty}(\Omega)\right)$. In this Note we first perform an analysis of the occurrence of free boundaries and their properties by means of some energy methods (see [2]) and afterwards we describe three uniqueness results obtained under suitable assumptions by using different techniques (see [1], [7], and [3]).

\section{Space localization results}

We assume throughout this section that $r(s):=|s|^{\alpha} \operatorname{sign}(s)$ with $\alpha \in(1, \infty)$. We define the local energy $D_{n}(P)$ of the density $n$ for a domain $P \subset \Omega \times(0, T)$ by:

$$
D_{n}(P)=\frac{1}{(\chi+1} \sup _{(\alpha, \tau) \in P}|n(x, \tau)|^{\alpha+1}+\int_{P}\left|\nabla n^{\alpha}\right|^{2} d x d \tau .
$$

Theorem 1. - (Finite speed of propagation) Let $x_{0} \in \Omega, 0<\rho_{0}<\operatorname{dist}\left(x_{o}, \partial \Omega\right)$, and $T>0$. Assume that $n_{1}=0, p_{1}=0$ in $B_{p_{o}}\left(x_{o}\right)$, and that

$$
R(u, v)\left(u^{\alpha}+v^{\alpha}\right) \geq-\kappa_{R}\left(u^{\alpha+1}+v^{\alpha+1}\right) \quad \text { for all } u, v \geq 0
$$

with $\kappa_{R} \geq 0$. Then, there exist $M>0$ and $T_{1} \in(0, T)$ such that, if $D_{n}\left(B_{\rho_{v}}\left(x_{o}\right) \times(0, T)\right)+$ $D_{p}\left(B_{\rho_{o}}\left(x_{o}\right) \times(0, T)\right) \leq M$, then $n(x, t)=0, p(x, t)=0$ for a.e. $x \in B_{\rho(t)}\left(x_{o}\right), t \in\left(0, T_{1}\right)$, where $\rho(t)=\rho_{0}-$ ct and $c>0$ is some constant independent of $t$.

For the next theorems we need a stronger condition on $R$, namely that for $\omega \subset \Omega$ and $b>0$, we have

$$
R(n(x, t), p(x, t)) \geq \ln (x, t)^{\delta} \quad \text { a.e. }(x, t) \in \omega \times(0, T), \quad \alpha+\delta<2 .
$$

THEOREM 2. - (Waiting time) Let $x_{0} \in \Omega, 0<\rho_{0}<\rho_{1}<\operatorname{dist}\left(x_{0}, \partial \Omega\right)$, and $T>0$. Assume that (5) holds for $\omega=B_{\rho_{0}}\left(x_{0}\right)$. Suppose furthermore that $\left\|n_{1}\right\|_{\alpha+1, B_{\rho}\left(x_{0}\right)}^{\alpha+1} \leq \varepsilon_{0}\left(\rho-\rho_{o}\right)_{+}^{\beta}$, where $\varepsilon_{0}>0$ and $\beta=(2 d(\alpha-1)+4(\alpha+1)) /(2(\alpha-1))>1$. Then there exist $M>0, \varepsilon_{1}>0$, and $T_{2} \in(0, T)$ such that, if $\varepsilon_{o} \leq \varepsilon_{1}$ and $D_{n}\left(B_{\rho_{o}}\left(x_{o}\right) \times(0, T)\right) \leq M$, then $n(x, t)=0$ for a.e. $x \in B_{\rho_{o}}\left(x_{o}\right), t \in\left(0, T_{2}\right)$.

THEOREM 3. - (Formation of vacuum) Let $\omega \subset \Omega \Omega$ be a domain, $x_{0} \in \omega$, and $T>0$. Assume that (5) holds. Then there exist $M>0, T_{3} \in(0, T), \sigma, \mu \in(0,1)$ such that if $D_{n}(\omega \times(0, T)) \leq M$, then $n(x, t)=0$ for a.e. $x \in B_{\rho(t)}\left(x_{0}\right), t \in\left(T_{3}, T\right)$, where $\rho(t)=\sigma\left(t-T_{3}\right)^{\mu}$.

The proofs of these theorems are based on local energy methods. For this we define the domain

$$
P=P(t)=\left\{(x, \tau) \in Q_{T}:\left|x-x_{o}\right| \leq \rho(\tau)=\rho+\sigma(\tau-t)^{\mu}, \tau \in(t, T)\right\} .
$$

We choose parameters $\rho, \sigma, t$, and $\mu$ as follows: Theorem 1: $P$ is a truncated cone with $\rho=\rho_{o}>0$, $\sigma=-c<0, t=0, \mu=1$; Theorem 2: $P$ is a cylinder $B_{\rho}\left(x_{o}\right) \times(0, \tau), 0<\rho \leq \rho_{o}, 0<\tau<T$; 


\section{J. I. Díaz, G. Galiano and A. Jüngel}

Theorem 3: $P$ is a paraboloid with $\rho=0, \sigma>0, \mu \in(0,1)$. The following integration by paris formula holds (see [2]):

$$
\begin{aligned}
& \frac{1}{\alpha+1} \int_{P \cap\{\tau=T\}} n(x, T)^{\alpha+1}+\int_{P}\left|\nabla n^{\alpha}\right|^{2} \\
& =\int_{\partial_{l} P}\left(\nabla n^{\alpha} \cdot \nu_{x}\right) n^{\alpha}-\frac{1}{\alpha+1} \int_{\partial_{l} P} \nu_{\tau} n^{\alpha+1}+\frac{1}{\alpha+1} \int_{P \cap\{\tau=0\}} n_{I}^{\alpha+1} \\
& \quad-\int_{P}\left(\frac{1}{\alpha} n \nabla V \cdot \nabla n^{\alpha}+n^{\alpha+1} \Delta V+R(n, p) n^{\alpha}\right)=I_{1}+\cdots+I_{4},
\end{aligned}
$$

where $\left(\nu_{x}, \nu_{\tau}\right)$ is the unitary exterior normal vector to the lateral surface $\partial_{l} P$.

Proof of Theorem 3. - We only show how to estimate $I_{4}$ since the remaining integrals can be estimated as in [2] by using an interpolation-trace lemma and Hölder's inequality. Using hypothesis (5) and Young's inequality, we get

$$
I_{4} \leq \frac{1}{2} \int_{P}\left|\nabla n^{\alpha}\right|^{2}+\left(c_{1} D_{n}(P)^{2-\alpha-\delta}-b\right) \int_{P} n^{\alpha+\delta}+c_{2}(T-t) \sup _{s \in(t, T)} \int_{P \cap\{\tau=s\}} n(x, s)^{\alpha+1},
$$

where $c_{1}, c_{2}>0$ depend on the local $L^{\infty}$-norms of $\nabla V, \Delta V$ respectively. Taking $\sigma>0$ small enough, we can ensure $P \subset \omega$. Choose $M>0$ small enough such that $c_{1} D_{n}(P)^{2-\alpha-\delta}<b / 2$, and let $T^{*} \in(0, T)$ be such that $c_{2}\left(T-T^{*}\right) \leq 1 / 2(\alpha+1)$. Then, for $T^{*}<t<T$,

$$
I_{4} \leq \frac{1}{2} \int_{P}\left|\nabla n^{\alpha}\right|^{2}-\frac{b}{2} \int_{P} n^{\alpha+\delta}+\frac{1}{2(\alpha+1)} \sup _{s \in(t, T)} \int_{P \cap\{\tau=s\}} n(x, s)^{\alpha+1} .
$$

The remaining nonnegative terms can be absorbed by the left hand side of (7). Now $I_{3}=0$, and we follow [2] to estimate $I_{1}$ and $I_{2}$, getting in this way a differential inequality for $E(t):=\int_{P}\left|\nabla n^{\alpha}\right|^{2}$ :

$$
E^{\gamma} \leq c\left(-\frac{d E}{d t}\right), \quad E(T)=0, \quad E(t) \leq D_{n}(P),
$$

where $t \in\left(T^{*}, T\right), c>0$, and $\gamma \in(0,1)$. For $M>0$ and $T-T_{3}>0$ small enough, we conclude that $E(t)=0$ for $t \in\left(T_{3}, T\right)$. This implies the assertion.

Proof of Theorem 2. - The integral $I_{4}$ is estimated as in the preceeding proof. Estimating as in [2], if $E(\rho):=\int_{P}\left(\left|\nabla n^{\alpha}\right|^{2}+\left|\nabla p^{\alpha}\right|^{2}\right)$, we get

$$
E^{1-1 / \beta} \leq c \frac{d E}{d \rho}+c\left(\varepsilon_{o}\right)\left(\rho-\rho_{o}\right)_{+}^{\beta-1}, \quad \beta>1 .
$$

Then, for global energies and $T-T_{2}>0$ small enough, we get $E\left(\rho_{\circ}\right)=0$.

Proof of Theorem 1. - Since we do not assume (5), we can rewrite $I_{4}$ :

$$
I_{2}+I_{4}=-\frac{\alpha}{\alpha+1} \int_{P} n^{\alpha+1} \Delta V-\frac{1}{\alpha+1} \int_{\partial_{l} P}\left(\nu_{\tau}+\nabla V \cdot \nu_{x}\right) n^{\alpha+1}-\int_{P} R(n, p) n^{\alpha} .
$$

Since $\nu_{\tau}+\nabla V \cdot \nu_{x} \geq\left(1+c^{2}\right)^{-1 / 2}\left(c+\nabla V \cdot e_{x}\right) \geq 0$ for sufficiently large $c>0$, where $e_{x}$ is the unit vector in the direction of $\nu_{x}$, we obtain

$$
I_{2}+I_{4}=T^{*} c_{3}(\Delta V) \sup _{s \in\left(0, T^{*}\right)} \int_{P \cap\{\tau=s\}} n(x, s)^{\alpha+1}-\int_{P} R(n, p) n^{\alpha} .
$$


Space localization and uniqueness of solutions of a quasilinear parabolic system...

A similar estimate holds for $p$. By adding the corresponding inequalities, we get

$$
-\int_{P} R(n, p)\left(n^{\alpha}+p^{\alpha}\right) \leq \kappa_{R} T^{*} \sup _{s \in\left(0, T^{*}\right)} \int_{P}\left(n^{\alpha+1}+p^{\alpha+1}\right) .
$$

Now proceed as in [2] to get a differential inequality similar to (9).

\section{Uniqueness of solutions}

We consider in this section a system slightly more general than (1) in which we include the case of a nonlinear transport term:

$$
(\mathcal{P}) \begin{cases}n_{t}-\operatorname{div}(\nabla r(n)+K(n) \nabla V)=F(n, p) & \text { in } Q_{T}, \\ p_{t}-\operatorname{div}(\nabla r(p)-K(p) \nabla V)=F(n, p) & \text { in } Q_{T}, \\ -\Delta V=p-n+C & \text { in } Q_{T},\end{cases}
$$

where $K \in \mathcal{C}^{1}(\mathbb{R})$. A similar system arises in two-phase filtrations (see [1]).

THEOREM 4. - Under one of the following set of conditions, the solution of $(P)$ is unique:

(i) $\left(K^{\prime}(s)\right)^{2} \leq M r^{\prime}(s)$ and $\left(\frac{\partial}{\partial s_{i}} F\left(s_{1}, s_{2}\right)\right)^{2} \leq M r^{\prime}\left(s_{i}\right), i=1,2$, for some $M>0$ with $s \in \mathbb{R}_{+}$.

(ii) $V \in L^{\infty}\left(0, T ; W^{2, \infty}(\Omega)\right), K(s)$ is linear, $\left|F\left(s_{1}, \sigma_{1}\right)-F\left(s_{2}, \sigma_{2}\right)\right| \leq c\left(\left|r\left(s_{1}\right)-r\left(s_{2}\right)\right|+\mid r\left(\sigma_{1}\right)-\right.$ $\left.r\left(\sigma_{2}\right) \mid\right)$ and

$$
\nabla V \cdot \nu=0 \quad \text { on } \Sigma_{D T} \text { for any third component } V \text { of the solution. }
$$

(iii) $n, p \in L^{1}\left(0, T ; W^{1, q}(\Omega)\right.$ ) (with $q=1$ if $N=1$ and $q>N$ if $N>1$ ) and $r \in C^{2}(0, \infty)$ is convex and strictly increasing.

Proof. - The proof of (i) follows arguments similar to [1]. For the proof of (ii), let $\left(n_{1}, p_{1}, V_{1}\right)$ and $\left(n_{2}, p_{2}, V_{2}\right)$ be two solutions and define $n:=n_{1}-n_{2}, p:=p_{1}-p_{2}$, and $V:=V_{1}-V_{2}$. Multiplying the two first equations satisfied by $(n, p, V)$ by appropriate test functions $\phi, \psi$, integrating by parts in $Q_{\tau}$ for $\tau \in(0, T)$, and adding the resulting integral identities, we obtain

$$
\begin{gathered}
\int_{0}^{\tau}\left(\left\langle n_{t}, \phi\right\rangle+\left\langle p_{t}, \psi\right\rangle\right)+\int_{Q_{\tau}}\left(\nabla\left(r\left(n_{1}\right)-r\left(n_{2}\right)\right) \cdot \nabla \phi+\nabla\left(r\left(p_{1}\right)-r\left(p_{2}\right)\right) \cdot \nabla \psi\right) \\
\quad=\int_{Q_{\tau}}\left(\nabla V \cdot\left(n_{1} \nabla \phi-p_{1} \nabla \psi\right)+\nabla V_{2} \cdot(n \nabla \phi-p \nabla \psi)\right)-\int_{Q_{\tau}}\left(R_{1}-R_{2}\right)(\phi+\psi),
\end{gathered}
$$

where $R_{i}=R\left(n_{i}, p_{i}\right)$. We choose $\phi, \psi$ as the unique solutions of

$$
-\Delta \phi=n(x, t), \quad-\Delta \psi=p(x, t) \quad \text { in } \Omega,
$$

with homogeneous mixed boundary conditions and for a.e. $t \in(0, T)$. Note that (14) implies that $\Delta V=\Delta(\psi-\phi)$. The term $\nabla V_{2} \cdot(n \nabla \phi)=-\left(\nabla V_{2} \cdot \nabla \phi\right) \Delta \phi$ can be estimated using (12) as in [7] to get

$$
-\int_{\Omega}\left(\nabla V_{2} \cdot \nabla \phi\right) \Delta \phi \leq c\left\|V_{2}\right\|_{W^{2, \infty}} \int_{\Omega}|\nabla \phi|^{2},
$$

and an analogous expression holds for $\int_{\Omega} \nabla V_{2} \cdot(p \nabla \psi)$. Since by (14), $2\left\langle n_{t}(\tau), \phi(\tau)\right\rangle=\|\nabla \phi(\tau)\|_{2}^{2}$, we obtain from (13) after standard estimations that

$$
\int_{\Omega}\left(|\nabla \phi(\tau)|^{2}+|\nabla \psi(\tau)|^{2}\right) \leq \int_{Q_{\tau}}\left(|\nabla \phi|^{2}+|\nabla \psi|^{2}\right) .
$$




\section{J. I. Díaz, G. Galiano and A. Jüngel}

From Gronwall's inequality and (14) we conclude the proof. The proof of (iii) uses a technique introduced in [3]. It is known (see [6] and [4]) that there exists a solution $\left(n_{\varepsilon}, p_{\varepsilon}, V_{\varepsilon}\right)$ to (11) with initial and boundary data $r\left(n_{D \varepsilon}\right)=r\left(n_{D}+\varepsilon e^{-\lambda_{1} t}\right), r\left(p_{D \varepsilon}\right)=r\left(p_{D}+\varepsilon e^{-\lambda_{1} t}\right), n_{I \varepsilon}=n_{I}+\varepsilon$, $p_{I \varepsilon}=p_{I}+\varepsilon$, with $\lambda_{1}, \varepsilon>0$ arbitrarily chosen, satisfying $n(x, t), p(x, t) \geq c \varepsilon e^{-\lambda t}>0$ in $Q_{T}$ for some $c>0$ and $\lambda>0$ independent of $r$ and $\varepsilon$. Moreover, such a solution converges strongly in $\left(L^{2}\left(Q_{T}\right)\right)^{3}$ (as $\left.\varepsilon \rightarrow 0\right)$ to a solution $\left(n_{1}, p_{1}, V_{1}\right)$ of $(11)$. Let $\left(n_{2}, p_{2}, V_{2}\right)$ be another solution of (1) and set $N_{\varepsilon}:=n_{\varepsilon}-n_{2}, P_{\varepsilon}:=p_{\varepsilon}-p_{2}, v_{\varepsilon}=: V_{\varepsilon}-V_{2}$. Define

$$
A_{n}^{\varepsilon}:=\frac{r\left(n_{\varepsilon}\right)-r\left(n_{2}\right)}{N_{\varepsilon}}, \quad \mathbf{B}_{n}^{\varepsilon}:=\frac{K\left(n_{\varepsilon}\right)-K\left(n_{2}\right)}{N_{\varepsilon}} \nabla w_{\varepsilon}, \quad R_{n}^{\varepsilon}:=\frac{R\left(n_{\varepsilon}, p_{\varepsilon}\right)-R\left(n_{2}, p_{\varepsilon}\right)}{N_{\varepsilon}},
$$

with similar definitions for terms involving $p$. Let $A_{n}^{\varepsilon, j}, \mathbf{B}_{n}^{\varepsilon, j}$, and $F_{n}^{\varepsilon, n}$ be smooth approximations of these functions. By the convexity of $r$ and the positivity of $n$, we have $A_{n}^{\varepsilon, j}>c(\varepsilon)$, with $\lim _{\varepsilon \rightarrow 0} c(\varepsilon)=0$. Also $B_{n}^{\varepsilon, j}$ and $R_{n}^{\varepsilon}$ are uniformly bounded in $\varepsilon$. Then, if $\psi, \xi, \eta$ satisfy

$$
\begin{cases}\psi_{t}+A_{n}^{\varepsilon, j} \Delta \psi+\mathbf{B}_{n}^{\varepsilon, j} \cdot \nabla \psi+R_{n}^{\varepsilon, j}(\psi+\xi)=0 & \text { in } Q_{T}, \\ \xi_{t}+A_{p}^{\varepsilon, j} \Delta \xi-\mathbf{B}_{p}^{\varepsilon, j} \cdot \nabla \xi+R_{p}^{\varepsilon, j}(\psi+\xi)=0 & \text { in } Q_{T}, \\ \Delta \eta=\operatorname{div}\left(p_{2} \nabla \xi-n_{2} \nabla \psi\right) & \text { in } Q_{T}, \\ \psi(x, T)=\chi_{n}^{\delta}(x), \quad \xi(x, T)=\chi_{p}^{\delta}(x) & \text { on } \Omega,\end{cases}
$$

with homogeneous mixed boundary conditions, we obtain

$$
\begin{gathered}
\int_{\Omega}\left(\chi_{n}^{\delta}(x) N_{\varepsilon}(x, T)+\chi_{p}^{\delta} P_{\varepsilon}(x, T)\right)=\varepsilon \int_{\Omega}(\psi(x, 0)+\xi(x, 0)) \\
-\int_{\Sigma_{D} T^{\prime}}\left[\left(r\left(n_{\varepsilon}\right)-r\left(n_{2}\right)\right) \nabla \psi+\left(r\left(p_{\varepsilon}\right)-r\left(p_{2}\right)\right) \nabla \xi\right] \cdot \nu
\end{gathered}
$$

As a consequence of the Alexandrov Maximum Principle and using the regularity $n, p \in$ $L^{1}\left(0, T ; W^{1, p}(\Omega)\right)$, we obtain $L^{\infty}$-uniform (in $\varepsilon$ ) estimates of $\psi$ and $\xi$. We now pass to the limit as $\varepsilon \rightarrow 0$ using a uniform boundary estimate (see [4], Lemma 2.10) to conclude that $\int_{\Omega}\left|N_{\varepsilon}(x, \tau)\right|+\left|P_{\varepsilon}(x, \tau)\right| \leq 0$

Note remise et acceptée le 26 mai 1997.

\section{References}

[1] Antontsev S. N., Díaz J. I. and Domansky A. V., 1993. Continuous dependence and stabilization of solutions of the degenerate system in two-phase filtration, Dinamika sploshnoi sredy, No. 107.

[2] Antontsev S., Díaz J. I., and Shmarev S., 1995. The support shrinking in solutions of parabolic equations with nonhomogeneous absorption terms, Ann. Fac. Sci. Toulouse, 4, pp. 5-30.

[3] Díaz J. I. and Kersner R., 1987. On a nonlinear degenerate parabolic equation in infiltration or evaporation through a porous medium, J. Differ. Equations, 69, pp. 368-403.

14] Galiano G., 1997. Sobre algunos problemas de la Mecánica de Medios Continuos en los que se originan fronteras libres, Tesis Doctoral, Universidad Complutense de Madrid, Madrid.

[5] Jüngel A., 1997. A nonlinear drift-diffusion system with electric convection arising in semiconductor and electrophoretic modeling, to appear in Math. Nachr.

[6] Jüngel A., 1996. Asymptotic analysis of a semiconductor model based on Fermi-Dirac statistics, Math. Meth. Appl. Sci., 19, pp. 401-424.

17] Rulla J., 1987. Weak solutions to Stefan problems with prescribed convection, SIAM J. Math. Anal., 18, pp. 1784-1800. 